\title{
Selective two-photon absorption in carbon dots: a piece of the photoluminescence emission puzzle
}

Carla, I. M. Santos, ${ }^{\text {a,b }}$ Inês F. A. Mariz, ${ }^{\mathrm{b}}$ Sandra N. Pinto, ${ }^{\mathrm{a}, \mathrm{c}}$ Gil Gonçalves, ${ }^{\mathrm{d}, \mathrm{e}}$ Igor Bdikin, ${ }^{\mathrm{d}}$ Paula A.A.P. Marques, ${ }^{\mathrm{d}}$ Maria Graça P.M.S. Neves, ${ }^{\mathrm{b}}$ José M. G. Martinho, ${ }^{\mathrm{a}}$ and Ermelinda M. S. Maçôas. *,a

Carbon nanodots (Cdots) are now emerging as promising nonlinear fluorophores for applications in biological environments. A thorough and systematic approach to the two-photon induced emission of Cdots that could provide design guidelines to control their nonlinear emission properties is still missing. In this work we address the nonlinear optical spectroscopy of Cdot prepared by controlled chemical cutting of graphene oxide (GO). The two-photon absorption in the 700-1000 nm region and the corresponding emission spectrum are carefully investigated. The highest two-photon absorption cross-section estimated was $130 \mathrm{GM}$ at $720 \mathrm{~nm}$. This value is comparable with the one reported for nanographenes with push-pull architecture. The emission spectrum dependes on the excitation mode. At the same excitation energy, nonlinear excitation results in an excitation-wavelength independent emission, while upon linear excitation the emission is excitation-wavelength dependent. The biphotonic interaction seems to be selective towards $\mathrm{sp}^{2}$ clusters bearing electron donor and acceptor groups found in push-pull architectures. Both linear and nonlinear emission can be understood based on the existence of isolated $\mathrm{sp}^{2}$ clusters involved in $\pi-\pi$ stacking interactions with clusters in adjacent layers. 


\section{Introduction}

Despite the ill-defined structure and the intense debate over the origin of their emission, the optical properties of carbon nanodots have been successfully explored in imaging, sensing, photocatalysis, optoelectronic devices and nanomedicine. ${ }^{1-4}$ In recent years, we have observed a rapidly growing interest in exploring the nonlinear emission of Cdots for application in biological environment due to their high two-photon absorption (TPA) cross-sections $\left(10^{4} \mathrm{GM}\right)$, combined with high luminescence quantum yields, water solubility, biocompatibility, photostability and versatile surface chemistry. ${ }^{5,}$ 6,7 Molecular fluorophores can be designed to have equally high TPA cross-sections. However, this is usually achieved at the expense of the emission quantum yields and water solubility. ${ }^{8} 9$ Strategies to improve the TPA cross-section in molecular fluorophores include the introduction of electron donor and acceptor groups to induce intramolecular charge transfer, extending the conjugation length between those groups by introducing a $\square$ conjugated bridge and improving the planarity of the $\pi$-system. ${ }^{10-12}$ A recently published review gives a good account of the latest developments concerning the two-photon induced emission in Cdots. ${ }^{13}$ There are many reports observing the twophoton emission of Cdots. However, the number of reports that validate the nonlinearity of the observed emission through its quadratic dependence on the excitation fluence is limited. This validation is necessary to eliminate artifacts such as interference from leaking second-order diffraction of the excitation light when broad-band light sources and diffraction gratings are used for excitation wavelength selection. If appropriate bandpath filters are not used, linear excitation in the UV/Vis due to leaking 2nd order diffraction at wavelength $\square / 2(350550 \mathrm{~nm})$ results in a much stronger emission signal than nonlinear excitation in the NIR at wavelength $\square(7001100 \mathrm{~nm}) .{ }^{12,13}$ Indeed, many of the early reports on upconverted emission attributed to anti-Stokes processes 
involving the $\square \square$ and $\square$ orbitals of carbene edge states are now believed to originate from such artifacts. In the literature on two-photon emission of Cdots, it is rare to find a two-photon absorption/excitation spectrum. ${ }^{14,15}$ The motivation for the present study is the limited quantitative data available on the TPA cross-section of Cdots and our superficial understanding of the phenomena. Table S1 in ESI compiles the quantitative data available on the TPA cross-section of Cdots. The results span a wide range of values from 22-50 000 GM. Such widely dispersed values are not at odds with the structural heterogeneity of Cdots as they can be produced by various synthetic approaches. The available data suggest that crystallinity and nitrogen doping are important factors in the optimization of the TPA cross-section. However, the limited number of reported TPA cross-section values and the scattering of absolute values fail to provide an accurate account of the importance of such factors. Most importantly, a recent study of Cdots produced by a variety of top-down and bottom-up methods shows that not all the Cdots have a measurable two-photon induced emission. ${ }^{16}$ This so far isolated report of

negative results raises the issue of biased literature data towards the best performing nonlinear Cdots due to the considerably larger impact of publishing positive results. A thorough and systematic approach to the two-photon induced emission of Cdots that could provide the design guidelines to control of their nonlinear emission properties is still missing. The lack of such guidelines is precluding the Cdots from achieving their full potential as nonlinearly responsive optical materials. Indeed, despite the many reports on the use of their linear emission in sensing applications ${ }^{2,17,18}$, the use of the analogous nonlinear emission for imaging and sensing is still in its infancy. ${ }^{7,19-22}$ The study of the origin of the linear emission of Cdots has been far more popular than that of their nonlinear optical spectroscopy. ${ }^{23,} 24$ The photophysical properties of 
nanosized graphene sheets have been theoretically predicted to be tuned by the size of the $\mathrm{sp}^{2}$ domains, edge configuration, shape, chemical functionalities, heteroatom doping and defects in the $\mathrm{sp}^{2}$ network. ${ }^{25}$ All these factors have been used to rationalize the optical properties of Cdots, but no general correlation with Cdots structure has been established. ${ }^{23,}{ }^{24}$ The ambiguity over Cdots emission is especially intriguing since the apparently high structural heterogeneity of the dots produced is not met by the similarities in their optical properties. Typically, two distinct regimes are observed in their emission spectrum, an excitation-independent one for short excitation wavelengths and an excitation-dependent one at longer excitation wavelengths. Cdots that have a carbogenic core, produced by solvothermal treatment of molecular carbon sources such as citric acid above $200^{\circ} \mathrm{C}$, and also from bulk carbon materials such as GO, are usually dominated by the excitation dependent regime. Cdots produced by solvothermal treatment of citric acid at lower temperatures are predominantly charatcerized by an excitation independent emission. The structural heterogeneity of the mixture seems to be the most trivial but not the most popular interpretation of this effect. ${ }^{24,26}$

The relevance of this work is to provide a thorough investigation of both linear and nonlinear optical properties of nitrogen-doped Cdots. The selectivity observed in the nonlinear spectroscopy of Cdots provides an extra piece of the puzzle, broadening our understanding of the nature of its emissive center.

\section{Experimental}

\subsection{Materials}

All chemicals used were of analytical pure grade. Graphite, ammonium hydroxide solution (28\% $\mathrm{NH}_{3}$ in $\mathrm{H}_{2} \mathrm{O}$ ) and hydrogen peroxide solution (30\% (w/w) in $\left.\mathrm{H}_{2} \mathrm{O}\right)$ were purchased from sigma Aldrich. 


\subsection{Synthetic procedure}

GO was prepared from natural graphite powder using the modified Hummers method. ${ }^{27}$ Briefly, $85 \mathrm{~mL}$ of $\mathrm{H}_{2} \mathrm{SO}_{4}$ was added under stirring to a mixture containing $2.5 \mathrm{~g}$ of graphite and $1.9 \mathrm{~g}$ of $\mathrm{NaNO}_{3}$ kept in an ice bath. After that, $11.25 \mathrm{~g}$ of $\mathrm{KMnO}_{4}$ was gradually added to the solution while stirring. After $2 \mathrm{~h}$, the resulting solution was removed from the ice bath, and the stirring was maintained for 5 days. The browncolored viscous slurry obtained was slowly added (ca. 1h) to $500 \mathrm{~mL}$ of an aqueous solution of $\mathrm{H}_{2} \mathrm{SO}_{4}$ (5 wt \%) under continuous stirring. The resulting mixture was maintained under stirring for another $2 \mathrm{~h}$. Subsequently, $10 \mathrm{~mL}$ of $\mathrm{H}_{2} \mathrm{O}_{2}$ was added to the mixture, stirred for further $2 \mathrm{~h}$ and then left to settle overnight. The mixture was filtered and the resulting solid was further purified by dispersion in $500 \mathrm{~mL}$ of an aqueous solution of $\mathrm{H}_{2} \mathrm{SO}_{4}$ (3 wt \%) and $\mathrm{H}_{2} \mathrm{O}_{2}$ (0.5 wt \%). After two days of precipitation, the supernatant solution was removed. This process was repeated five times. The solid product obtained after the rigorous cleaning process was rinsed using copious amounts of distilled water. Finally, the resulting GO was dried by lyophilization.

The preparation of the Cdots was based on the hydrothermal treatment of GO in the presence of $\mathrm{NH}_{4} \mathrm{OH}$ and $\mathrm{H}_{2} \mathrm{O}_{2}$ described in the literature. ${ }^{28}$ Three batches were prepared (b1, b2 and b3), where the $\mathrm{GO}$ and the $\mathrm{NH}_{4} \mathrm{OH}$ in the reaction mixture were fixed (120 mg of $\mathrm{GO}$ and $1.5 \mathrm{~mL}$ of $\mathrm{NH}_{4} \mathrm{OH}$ ), and the $\mathrm{H}_{2} \mathrm{O}_{2}$ was systematically increased by twofold from 1.5 (b1), 3.0 (b2) to $6 \mathrm{~mL}$ (b3). The overall reaction volume was kept constant at $20 \mathrm{~mL}$ by the addition of deionized water. In the experimental procedure, the GO was first dispersed in deionized water by mechanical stirring. Subsequently, $\mathrm{NH}_{4} \mathrm{OH}$ and $\mathrm{H}_{2} \mathrm{O}_{2}$ were added to the dispersion and the mixture was further stirred for $10 \mathrm{~min}$ at room temperature. The mixture was transferred to a Teflon lined autoclave and heated at 
$150{ }^{\circ} \mathrm{C}$ for $5 \mathrm{~h}$. After cooling to room temperature, the black precipitate was filtered out using a $0.02 \mu \mathrm{m}$ anopore inorganic membrane (AnodiscTM, Watman). The filtrate was stirred and heated at $100{ }^{\circ} \mathrm{C}$ for $1 \mathrm{~h}$ to remove any remaining $\mathrm{NH}_{3}$. The light-yellow supernatant was dialyzed in a $1 \mathrm{KDa}$ dialysis bag against deionized water for 7 days. The resulting material was lyophilized and the overall yields varied from 30-40 wt\%.

\subsection{Chemical and structural properties}

The chemical and structural properties of the Cdots were characterized by transmission electron microscopy (TEM), atomic force microscopy (AFM), Fourier transform infrared (FTIR), X-Ray photoelectron spectroscopy (XPS) and confocal Raman microscopy (Raman). XPS spectra were acquired in an Ultra High Vacuum (UHV) system with a base pressure of $2 \times 10^{-10}$ mbar located at TEMA, University of Aveiro. The system is equipped with a hemispherical electron energy analyzer (SPECS Phoibos 150), a delay-line detector and a monochromatic AlKa (1486.74 eV) X-ray source. High resolution spectra were recorded at normal emission take-off angle and with a passenergy of $20 \mathrm{eV}$ for C1s and N1s spectra. The FTIR spectra were recorded from $\mathrm{KBr}$ pellets (Aldrich, 99\%, FT-IR grade) using a Mattson 7000 FT-IR spectrometer by accumulating 256 interferograms with a resolution of $8 \mathrm{~cm}^{-1}$. Atomic force microscopy (NanoScope IV SPM Controller, Veeco) in tapping mode was used for surface topography studies on the C-dots deposited on mica substrate. Raman characterization was carried out by unpolarized Raman microscopy (Horiba LabRAM HR 800 Evolution) using a $633 \mathrm{~nm}$ diode laser delivering $10 \mathrm{~mW}$ at the sample position and a 600 groves $\mathrm{mm}^{-1}$ grating. Data were collected in the range of $900-1800 \mathrm{~cm}^{-1}$ with a resolution of $4 \mathrm{~cm}^{-1}$ using only $10-20 \%$ of the laser power, with acquisition times of $20 \mathrm{~s}$ and 20 accumulations, and using either 50x or 100X objective lenses. Transmission electron microscopy (TEM) images were obtained on a Hitachi transmission electron 
microscope (Model H-8100 with a LaB6 filament) with an acceleration voltage of 200 $\mathrm{kV}$. One drop of the dispersion of the C-dots in water was deposited on the TEM grid (ultrathin carbon grid on a lacey carbon support film, 400 mesh (PELCO®), and dried under a flow of dried nitrogen. The images were processed with the Fiji software to calculate the size distribution plots from the Feret diameter.

\subsection{Optical properties}

The optical properties were characterized by Ultraviolet-Visible (UV-Vis) linear absorption spectroscopy, steady-state and transient fluorescence spectroscopy, and twophoton emission spectroscopy.

The linear absorption spectra were recorded in a JASCO V-540 spectrophotometer. The fluorescence spectra were recorded using a Horiba Jobin Yvon Fluorlog 3-22 Spectrofluorimeter with a $450 \mathrm{~W}$ xenon lamp. The fluorescence quantum yields were determined by the reference method using Fluorescein ( $\left.\mathrm{pH} 11, \phi=0.92, \lambda_{\mathrm{em}}=500-560\right)$ as standard. ${ }^{29}$ The fluorescence decay curve were measured in $5 \mathrm{~mm}$ quartz cuvettes by the Single-Photon Timing technique under excitation at $330 \mathrm{~nm}$. The excitation source was the second harmonic of a Coherent Radiation Dye laser 700 series (laser dye DCM, 610-680 nm, $130 \mathrm{~mW}, 5 \mathrm{ps}, 4 \mathrm{MHz}$ ). The emission was collected at the magic angle using a Jobin Yvon HR320 monochromator (Horiba Jovin Ivon Inc.). The instrument response functions for deconvolution (35-80 ps FWHM) were generated by scattering dispersions of colloidal silica in water. The solutions were kept under gentle stirring during the data collection. Blank decays were acquired to ensure that dark photon counts were negligible. Decay curves were stored in 1024 channels with 48.8 ps per channel and an accumulation of 20k counts in the peak channel. The fluorescence decays were analyzed by a non-linear least-squares reconvolution method using the TRFA DP software by SSTC (Scientific Software Technologies Center, Belarusian 
State University, Minsk, Belarus). The two-photon absorption (TPA) spectra were measured by two-photon induced fluorescence (TPF) using Fluorescein at $\mathrm{pH} 11$ as reference to account for collection efficiency and pulse characteristics. ${ }^{30}$ A modified setup that follows the one described by $\mathrm{Xu}$ and Webb was used to estimate the TPA cross-section in the $710-990 \mathrm{~nm}$ region. ${ }^{31}$ The excitation source was a Ti:sapphire laser (Tsunami BB, Spectra-Physics, 710-990 nm, 1.7 W, 100 fs, 82 MHz). The two-photon emission was measured within a narrow wavelength bandwidth selected by the H20Vis Jobin Yvon monochromator placed at the entrance of a PMC-100-4 photomultiplier (Becker and Hickl GmbH). The integrated TPF over the entire emission band was extrapolated using the emission spectra corrected by the detector sensitivity. The twophoton absorption cross-section was calculated from the equation:

$\sigma_{2}(\lambda)=\left(\frac{F_{2}(\lambda)}{\phi C n}\right)_{s} k_{e f f}(\lambda)$

$k_{e f f}(\lambda)=\left(\frac{\phi C n \sigma_{2(\lambda)}}{F_{2}(\lambda)}\right)_{r e f}$

where $F_{2}$ stands for integrated fluorescence intensity, $\phi$ is the one-photon excited fluorescence quantum yield, $n$ refers to the refractive index in solution, $C$ is the concentration in mol/dm ${ }^{3}$ and $s$ and ref are relative to the sample and the TPA reference, respectively. $k_{\text {eff }}$ is an excitation wavelength dependent correction factor that depends on the pulse properties. ${ }^{30}$ The two-photon absorption (TPA) and two-photon excitation (TPEx) spectra are the plots of $\sigma_{2}(\lambda)$ and $F_{2}(\lambda) k e f f(\lambda)$ as a function of the excitation wavelength, $\square$, respectively To calculate $\sigma_{2}(\lambda)$ we need to estimate the concentration of the dispersion, and for that we need to know the molecular weight. The molecular weight was estimated assuming a cilindrical shape with a mean diameter given by TEM, the average height given by AFM and the density of the graphite $\left(2.25 \mathrm{~g} / \mathrm{cm}^{3}\right)$. The emission intensity dependence of the excitation power was confirmed to be quadratic. 


\subsection{Cellular internalization protocol}

HEK 293 T and MCF7 cells were cultured and maintained in DMEM with 10\% FBS (ThermoFisher Scientific) and 1\% of penicillin-streptomycin (Sigma Chemical Co., St. Louis, $\mathrm{MO})$ at the incubator with controlled temperature $\left(37^{\circ} \mathrm{C}\right)$, humidity and $\mathrm{CO}_{2}$ levels (5\%). The cells were seeded on sterilized tissue culture treated wells ( $\mu$-Slide 8 well, glass bottom, Ibidi GmbH, Munich) for up to $48 \mathrm{~h}$. After cell confluency reaches $\sim 70 \%$, an aqueous dispersion of Cdots from batch 3 was added to each well to obtain a final concentration of $35-70 \mu \mathrm{g} / \mathrm{ml}$ (dilutions were made in cell medium). To remove the unbound C-dots a washing step with Dulbecco's phosphate-buffered saline (DPBS, Thermo Fisher Scientific) was included. Alternatively, after incubation with C-dots, both cells lines were stained with WGA-Alexa Fluor 594 and Hoechst 33342 according to the instructions of the supplier (Thermo Fisher Scientific, Image-iTs LIVE Plasma Membrane and Nuclear Labeling Kit). A final washing step with DPBS was also included. The cells were imaged using a laser scanning confocal microscope (Leica TCS-SP5) equipped with a continuous Ar-ion laser (Multi-line LASOSs LGK 7872 ML05) and a Ti:sapphire laser (Spectra-Physics Mai Tai BB, 710-990 nm, 100 fs, 82 MHz). A 63x 1.2 N.A. water immersion objective was used (HCX PL APO CS 63.0x 1.20WATERUV).

\section{Results and discussion}

\subsection{Chemical and structural properties}

The synthetic procedure followed closely the one described in the literature for the large-scale preparation of graphene quantum dots (GQDs) based on the solvothermal treatment of GO in the presence of $\mathrm{H}_{2} \mathrm{O}_{2}$ and $\mathrm{NH}_{4} \mathrm{OH}^{28}$ The amounts of $\mathrm{GO}$ and $\mathrm{NH}_{4} \mathrm{OH}$ were kept constant and the added $\mathrm{H}_{2} \mathrm{O}_{2}$ was systematically increased by two- 
fold from 1.5, 3.0 to $6 \mathrm{~mL}$ in three different batches (b1, b2 and b3, respectively). These reaction conditions were designed aiming at the production of Cdots with different ratios of nitrogen containing functional groups. It is known that the production of Cdots by solvothermal treatment with ammonia alone results almost exclusively in functionalization of the Cdots with primary amines. ${ }^{32}$ On the other hand, the solvothermal treatment with ammonia in the presence of $\mathrm{H}_{2} \mathrm{O}_{2}$ leads to nearly equal amounts of amine and amide groups on the dots. ${ }^{28}$ The strong oxidizing effect of $\mathrm{H}_{2} \mathrm{O}_{2}$ results in the formation of carboxylic groups upon reaction with the epoxides in the GO, which in turn will react with the $\mathrm{NH}_{4} \mathrm{OH}$ at high temperatures to produce amides. Thus, it was hypothesized that the amide/amine ratio could be tuned by varying the concentration of $\mathrm{H}_{2} \mathrm{O}_{2}$. Nevertheless, even though both the FTIR and XPS data in
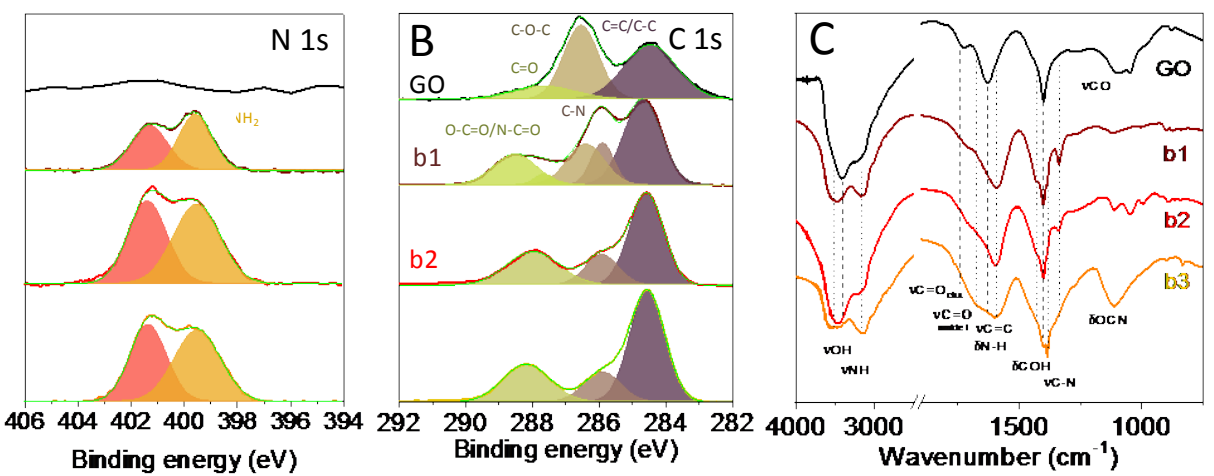

Figure 1 show that the amount of $\mathrm{H}_{2} \mathrm{O}_{2}$ used in the chemical cutting of GO affected the composition of the final material, the produced Cdots did not have an amide to amine functionality ratio significantly different from each other.

Fig. 1 Characterization of the Cdots and the starting GO by X-Ray photoelectron spectroscopy (A and B) and Fourier Transform Infrared Spectroscopy (C). The b1, b2 and b3 labels refer to Cdots produced with increasing concentration of $\mathrm{H}_{2} \mathrm{O}_{2}$. (A) $\mathrm{N} 1 \mathrm{~s}$ spectra showing the new bands due to the amide (401.3 eV) and amine (399.5 eV). (B) High resolution $\mathrm{C}$ 1s spectra showing the decrease in intensity of the band assigned to 
the epoxide (286.5 eV) in the dots. (C) FTIR spectrum highlighting the new bands due to the functionalization by nitrogen containing groups (dotted line), and the GO bands whose intensity decreases in the Cdots or are overshadowed by new bands (dashed line).

This is particularly evident from the XPS data (high resolution data in Figure $1 \mathrm{~A}$ and $1 \mathrm{~B}$, and wide-scan in Figure S1 of ESI). In the Cdots, new bands appear in the $\mathrm{N}$ 1s region ( $400 \mathrm{eV})$ assigned to nitrogen containing functional groups. The bands at $399.5 \mathrm{eV}$ and $401.3 \mathrm{eV}$ can be assigned to primary amines and amides, respectively. Based on the integrated intensity of the aminic and amidogenic nitrogen in the high resolution XPS spectra, the amine/amide intensity ratio is close to 1 in all the three batches. Table

S2 in ESI shows the calculated atomic percentage composition for carbon and nitrogen of the dots and of the starting GO. The atomic percentage of nitrogen increases with the concentration of $\mathrm{H}_{2} \mathrm{O}_{2}$ from b1 (4\%) to b2 (10\%) and it is not affected significantly by increasing further the concentration of $\mathrm{H}_{2} \mathrm{O}_{2}$ from b2 to b3 (8\%). In the C1s region, the high resolution XPS spectrum of GO shows the characteristic peaks of $\mathrm{sp}^{3} / \mathrm{sp}^{2}$ carbon at lower biding energies (284.5 eV), the epoxy (C-O-C) and hydroxylic (C-OH) both contributing to the shoulder at $286.5 \mathrm{eV}$, and the carbonyl $(\mathrm{C}=\mathrm{O})$ at higher binding energies $287.2 \mathrm{eV}$. In the Cdots the band at $286.5 \mathrm{eV}$ decreases and shifts to lower energies due to the replacement of epoxides by nitrogen containing functional groups. The epoxide band is stronger in b1 than in b2 or b3, which agrees with the lower degree of functionalization of b1 by nitrogen containing group. The band at at 288.5 has contributions from oxidized carbon $(\mathrm{O}-\mathrm{C}=\mathrm{O} / \mathrm{COOH})$ and $\mathrm{N}-\mathrm{C}=\mathrm{O}$ of amides. The Cdots in b2 reproduce the $\mathrm{H}_{2} \mathrm{O}_{2}: \mathrm{NH}_{4} \mathrm{OH}$ weight ratio used in ref ${ }^{28}$ and the spectroscopic data (FTIR, XPS and UV-Vis absorption and emission) show that the final product is similar 
to the yellow dots obtained earlier. The only appreciable difference is that the Cdots in b2 have as many amines as amide groups (N/C of $8.4 \%$ for amine and 7.7\% for amide) whereas the yellow dots were reported to be slightly off balanced towards the amine functionality (N/C of $12 \%$ for amine and $10 \%$ for amide).

The FTIR spectra of the Cdots is compared with that of GO in Figure 1C, giving support to the presence of nitrogen containing functional groups in the Cdots. The FTIR spectrum of the starting GO shows the typical features of oxygen-containing functional groups, such as the hydroxyl stretching $\left(\square \mathrm{OH}\right.$ ) at $3400 \mathrm{~cm}^{-1}$, the carbonyl stretching ( $\square \mathrm{C}=\mathrm{O}$ ) at $1730 \mathrm{~cm}^{-1}$, the $\mathrm{COH}$ bending $\left(\square \mathrm{COH}\right.$ ) at $1400 \mathrm{~cm}^{-1}$ and the C-O stretching ( $\square \mathrm{CO})$ at $1140-1020 \mathrm{~cm}^{-1}$. The $\mathrm{C}=\mathrm{C}$ stretching $(\square \mathrm{C}=\mathrm{C})$ appears at $1630 \mathrm{~cm}^{-1} .33$ The presence of amides in the Cdots results in new bands due to the amide I ( $\square \mathrm{C}=\mathrm{O}$ ) and II ( $\square \mathrm{NH}$ ) modes that increase the broadness of the band envelope in he $1700-1500 \mathrm{~cm}^{-1}$ region. The $\square \mathrm{N}-\mathrm{H}$ mode of both amines and amides can be responsible to the evident change in the relative intensity of the broad bands at $3500-3000 \mathrm{~cm}^{-1}$. The shoulder at $1430 \mathrm{~cm}^{-1}$ and the sharper feature at $1380 \mathrm{~cm}^{-1}$ can be assigned to amide III bands.

Since the amide/amine functionality ratio did not depend on the reaction conditions nor were the optical properties significantly affected (to be discussed later), the following discussion of the structural and optical properties of Cdots will be focused on only one of the batches, b3. In Figure 2 illustrative TEM and AFM images of b3 are shown together with the Raman spectrum of the Cdots and the starting GO. The TEM image shows a uniform size distribution centered around $4 \mathrm{~nm}$. Based on the AFM data the maximum height of the dots is $3.7 \mathrm{~nm}$ and their average height is $1.5 \mathrm{~nm}$. The Raman spectra are dominated by the $\mathrm{D}$ and $\mathrm{G}$ bands characteristic of polyaromatic hydrocarbons confirming the existence of extended $\mathrm{sp}^{2}$ clusters in the Cdots. ${ }^{34}$ The broadness of the bands is attributed to the structural heterogeneity of the material. The 
$\mathrm{G}$ band relates to the collective $\mathrm{C}=\mathrm{C}$ stretching within the $\mathrm{sp}^{2}$ cluster and the $\mathrm{D}$ band is associated with the ring breathing mode. The intensity ratio of these two bands $\left(\mathrm{I}_{\mathrm{D}} / \mathrm{I}_{\mathrm{G}}\right)$ has been previously correlated with the size of nanographene clusters within carbonbased materials. Following the Tuinsta and Köning relationship derived for nanocrystalline graphite, where the $\mathrm{I}_{\mathrm{D}} / \mathrm{I}_{\mathrm{G}}$ is inversely proportional to the $\mathrm{sp}^{2}$ cluster size, the average cluster size of $\mathrm{GO}$ is $4 \mathrm{~nm}\left(\mathrm{I}_{\mathrm{D}} / \mathrm{I}_{\mathrm{G}}=1.2\right)$ decreasing to $3 \mathrm{~nm}\left(\mathrm{I}_{\mathrm{D}} / \mathrm{I}_{\mathrm{G}}=1.5\right)$ in the Cdots. ${ }^{35}$ For the Cdots, a smaller cluster size of ca. $1.5 \mathrm{~nm}$ would be expected from the Ferrari and Robertson relationship derived for amorphous carbon, where the $I_{D} / I_{G}$ is proportional to the square of the cluster size. ${ }^{36}$ Given the lateral size distribution of the dots peaking at $4 \mathrm{~nm}$ both values are equally possible. Noteworthy is the fact that the existence a non-negligible amount of isolated small molecules in more amorphous domains cannot be ruled out based on the absence of other molecular vibrations in the Raman
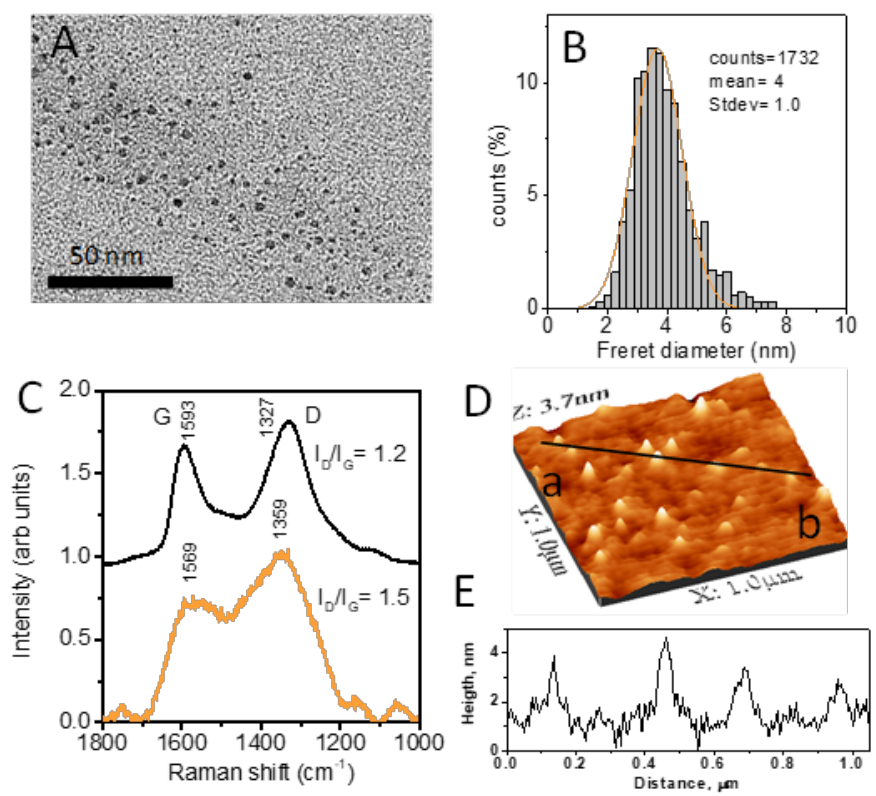

Fig. 2 Characterization of the Cdots in batch 3 by TEM (A and B), Raman spectroscopy (C) and AFM (D). (A) The TEM image and (B) the size distribution show that the diameter is centered at $4 \mathrm{~nm}$. (C) The Raman spectrum of the Cdots is shown in 
comparison with that of the starting GO. (D) The AFM image shows that maximum height of the dots is $3.7 \mathrm{~nm}$. (E) Cross-section of AFM image (D) along the ab line.

spectra in addition to the dominant $\mathrm{D}$ and $\mathrm{G}$ bands. Unlike isolated molecular vibrations, the $\mathrm{D}$ and $\mathrm{G}$ lattice modes are easily recognized despite the fluorescence background in the Raman spectra due to their intensity enhancement by strong electronphonon coupling. Thus, while the Raman spectrum confirms the existence of extended $\mathrm{sp}^{2}$ clusters it only gives an upper limit to the cluster size. No significant information about the relative concentration of nanographene domains and more amorphous domains can be obtained.

From the chemical and structural characterization discussed above, the Cdots produced could be classified as either carbon nanoparticles (CNP), that are quasispherical particles composed of polycrystalline nanographene clusters, or GQD that have a similar morphology but are usually pictured as multilayer stacks of $\mathrm{sp}^{2}$ hybridized carbon. ${ }^{23}$ The boundaries between these two types of dots are not sharp.

\subsection{Optical properties}

\subsubsection{Linear optical properties}

The photophysical properties (absorption, emission and photoexcitation spectra, fluorescence decay curves and quantum yields) are similar for all batches of produced Cdots. Figure 3 shows the main results forbatch b3, while corresponding results for b1 and b2 are shown in Figure S2 of the ESI. The linear optical properties of these nitrogen doped Cdots are similar to many other Cdots produced from GO. ${ }^{7}, 34$, 37-41 In general, these dots show an absorption extending from the red part of the visible spectra into the ultraviolet (UV) (Figure 3A). In all the Cdots it was possible to distinguish some overlaying structure in the visible part of the absorption spectrum. The emission spectrum is excitation-wavelength-dependent with the strongest emission peaking at 
$510 \mathrm{~nm}$ upon excitation at $260 \mathrm{~nm}$ (Figure3B) due to the strong absorption of the Cdots in the UV. The ensemble quantum yield increases towards the long excitation wavelengths from $3 \%$ to $11 \%$ (excitation at $350-460 \mathrm{~nm}$ ) stabilizing at $10 \%$ above 480 $\mathrm{nm}$ (Figure S3 in ESI). The contribution of different emitting sites to the absorption signals can be isolated by collecting the photoexcitation spectra at different emission wavelengths (Fig. 3C).

In the excitation-wavelength dependence of the emission spectrum it is possible to distinguish two different regions (Figures 3D and 3E): Region I for excitation above $350 \mathrm{~nm}\left(\lambda_{\mathrm{exc}}>350 \mathrm{~nm}\right)$, where the emission maxima shifts towards longer wavelengths and the FWHM first decreases and later (above $450 \mathrm{~nm}$ ) has a modest increase with the excitation wavelength; and Region II for excitation below $350 \mathrm{~nm}\left(\lambda_{\text {exc }}<350 \mathrm{~nm}\right)$, where the position of the emission maxima is excitation-independent and the FWHM increases with the excitation wavelength.
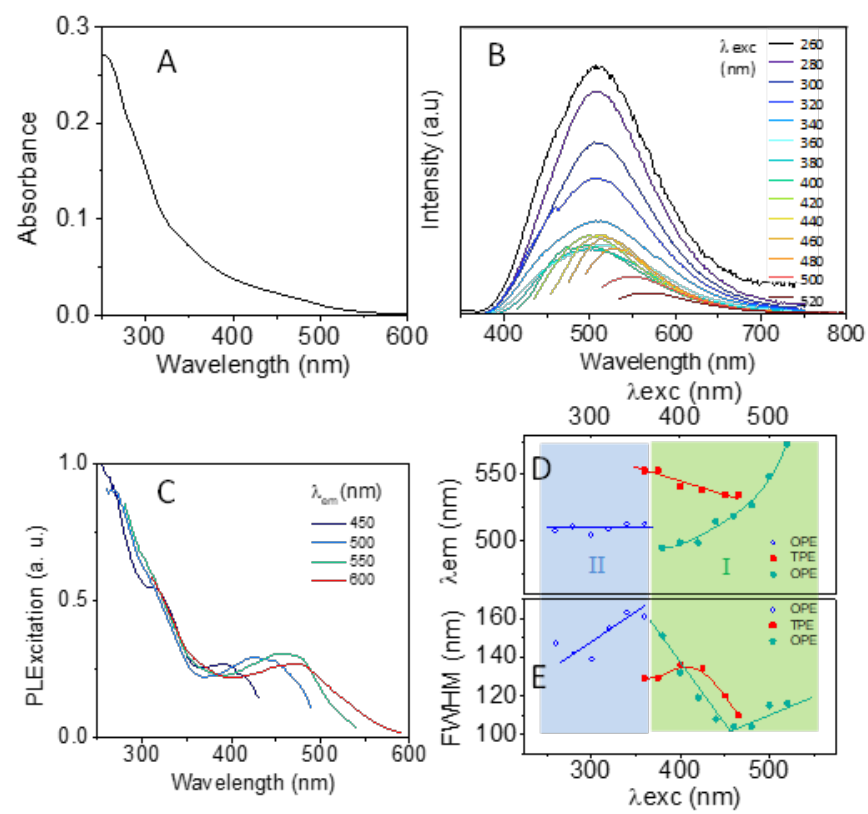

Fig. 3 Optical properties of Cdots: (A) Absorption spectrum. (B) Emission spectrum measured at different excitation wavelength $\left(\lambda_{\text {exc }}, \mathrm{nm}\right)$. (C) Photoluminescence excitation spectra collected at different emission wavelength $\left(\lambda_{\mathrm{em}}, \mathrm{nm}\right)$. (D) Excitation- 
wavelength dependence of the emission maxima and (E) FWHM of the emission band with region I and region II discussed in the text indicated in blue and green, respectively. In plots $\mathrm{D}$ and $\mathrm{E}$, the trend for the two-photon emission (TPE) is also indicated in red to allow for a direct comparison with one photon emission (OPE).

Excitation dependent emission $\left(\lambda_{\text {exc }}>350 \mathrm{~nm}\right)$

For $\lambda_{\text {exc }}>350 \mathrm{~nm}$, the emission is strongly excitation-dependent (Figure 3B). The FWHM of the emission band decreases while the emission maxima shift to longer wavelengths with the excitation wavelength (Figures 3D and 3E). The photoexcitation spectra collected at different emission wavelengths (450 $\mathrm{nm}<\lambda_{\text {em }}<600 \mathrm{~nm}$ ) show welldefined excitation bands in the visible

peaking between 400-500 nm (Figure 3C). The mirror image relationship between the photoexcitation and corresponding emission spectrum (Figure S4 in SI) and the small Stokes-shift strongly support the localization of the excitation and energy relaxation process in the same site, excluding the possibility of inter-site charge and energy transfer effects. The experimental data support the assignment of the emitting site to isolated $\mathrm{sp}^{2}$ clusters within the Cdots. The $\pi$ - $\pi$ stacking between $\mathrm{sp}^{2}$ clusters in consecutive layers can lead to broad unstructured absorption and emission bands like those observed in crystals of polyaromatic hydrocarbons (PAHs). ${ }^{42}$ Indeed, the typical inter-layer distances found in Cdots $(0.33 \mathrm{~nm})$ are similar to the separation between monomers in crystals of disk-shaped PAHs such as pyrene, perylene, coronene and ovalene. ${ }^{42}$ In such PAHs crystals, the lattice unit is composed by a pair of almost completely overlapping parallel molecules with an interplanar distance of $0.35 \mathrm{~nm} .^{42}$ Broad absorption and emission bands result from the shallow potential of the dimer in the unit cell. 
The fact that all the photoexcitation and emission spectra show similar features although shifted in energy (linear dependence of the emission maxima on the excitation wavelength) suggest that there are certain structural similarities between the emissive sites. This smooth modulation of the optical properties can be due to the chemical environment (defects imposing different degrees of distortion from planarity, presence of amines) or to small differences in the $\mathrm{sp}^{2}$ cluster structure (number of benzene rings or shape). In general, bigger clusters have smaller energy gaps. If the $\mathrm{sp}^{2}$ clusters have a distribution of sizes that closely mimics the smooth distribution of sizes of the host dots, then the redshift of the emission maxima with the excitation wavelength would be a trivial consequence of the size distribution of the clusters. In addition, within a particular cluster the functionalization with amines extends the $\pi$-conjugated system and promotes the charge-transfer from the amine group to the $\pi$-conjugated system.

The fluorescence decay curves measured in the 450-600 $\mathrm{nm}$ region upon excitation at $330 \mathrm{~nm}$ are multiexponential. They have small and non-monotonic changes in the average lifetime that tend to peak around the maximum wavelength of emission $\left(\tau_{\text {aver }}=\right.$ 1.5-3.0 ns, Figure S3 in SI). A similar correlation between the emission wavelength and photoluminescence lifetime has been reported earlier and interpreted on the basis of a combination of effects, including the size distribution of the dots and the functionalization by nitrogen-containing functional groups. ${ }^{43}$

Excitation independent emission $\left(\lambda_{\text {exc }}<350 \mathrm{~nm}\right)$

The excitation-independent emission in region I is a trivial effect of the simultaneous excitation of all the emitting clusters. This is clearly illustrated in Figure 3C, where the photoexcitation spectra collected at different emission wavelengths overlap below 350 nm. Thus, the emission spectrum should be a contribution from all the different types of 
emitting sites with the maxima determined by the most abundant emitting site or the most efficient emitter.

In this excitation region the resulting emission band has its maximum intensity fixed at $510 \mathrm{~nm}$ but it displays a shoulder of varying intensity at shorter wavelengths $\left(\lambda_{\mathrm{em}} \approx\right.$ $450 \mathrm{~nm}$ ) (Figure 3B). In fact, all the Cdots produced in this work have some contribution from a band peaking at shorter wavelengths (445 $\mathrm{nm})$. In the following discussion this is labeled as the blue emitting site. As shown in detail in Figure 4, this band is more intense upon excitation at $\lambda_{\mathrm{ex}}=320 \mathrm{~nm}$ and its contribution is stronger in b1. The blue emission is assigned to a nitrogen-free site based on the comparison of the UV-Vis spectroscopic data of nitrogen-doped Cdots with those of undoped Cdots obtained from a control batch (Figure 4). The emission spectrum of the undoped Cdots is largely dominated by a narrow emission band peaking at $430 \mathrm{~nm}$ (FWHM of $70 \mathrm{~nm}$ ) with a corresponding photoexcitation maximum at $315 \mathrm{~nm}$. This control batch was produced by subjecting the GO to the same hydrothermal treatment used in b1-b3 without adding $\mathrm{H}_{2} \mathrm{O}_{2}$ and $\mathrm{NH}_{4} \mathrm{OH}$. The size distribution peaking at $3 \mathrm{~nm}$ and photophysical properties of the undoped Cdots are summarized in Figure S5 of the SI. The higher intensity of the blue emission in b1 when compared with b2 and b3 correlates well with its lower atomic percentage of nitrogen (Table S2 in ESI). Due to the overlap of the blue emission with the photoexcitation spectra of b1-b3, energy transfer from nitrogen-free sites to nitrogen-dopped sites would be possible as long as they coexist in the same Cdot. However, the lack of a band at $320 \mathrm{~nm}$ in the photoexcitation spectra of b1-b3 collected in the 500-600 nm region (see Figure 3 and Figure S4) does not support the existence of an energy transfer process. 


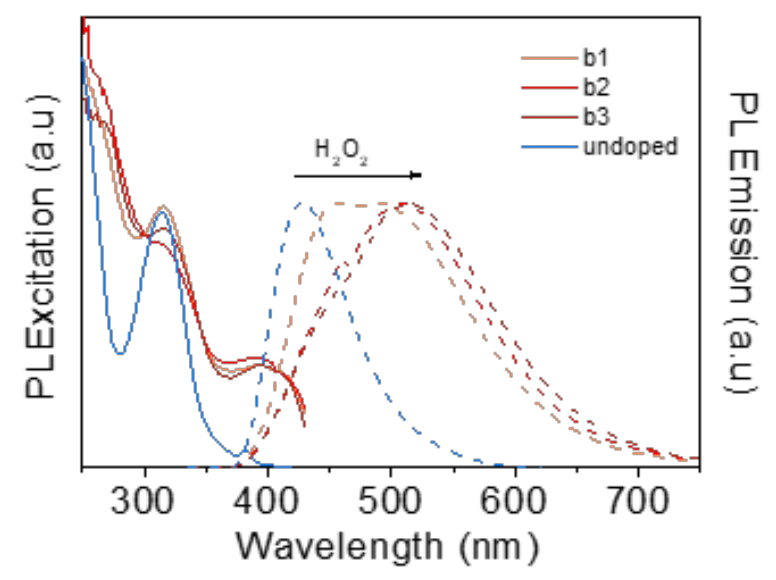

Fig. 4 Comparison between the emission spectra of the Cdots excited at $320 \mathrm{~nm}$ (dashed line) and of their photoexcitation spectra collected at $450 \mathrm{~nm}$ (straight line) with the corresponding spectra recorded in the undoped dots. All the spectra are normalized by their maximum intensity.

The spectroscopic features of the undoped Cdots and the blue emitting site in b1-b3 (absorption and emission maxima, large Stokes shift and excitation wavelength independence) are strikingly similar to those of blue emitting bottom-up dots produced from citric acid. ${ }^{44}$ The molecular origin of nitrogen doped Cdots produced from citric acid in the presence of different amines is currently the subject of debate. ${ }^{45-47}$ It has been argued that in such dots, small molecular fluorophores embedded in a matrix of $\mathrm{sp}^{3}$ hybridized carbon are responsible for the blue emission at 440-470 nm. Citrazinic acid derivatives on the edge of Cdots have been pointed out as responsible for the excitationwavelength independence and large Stokes shift of their blue emission. In undoped Cdots, the formation of citrazinic acid derivatives is unlikely due to the lack of a nitrogen source in the reaction mixture. Alternatively, in a recent work, the dominant fluorophore in blue emitting bottom-up Cdots was identified as pyrene. ${ }^{44}$ The assignment was based on the broad and structureless emission band of the pyrene 
excimers that contrast with the typical vibronic structure of emission of isolated PAHs. $^{48}$

\subsubsection{Nonlinear optical spectroscopy}

The nonlinear spectroscopic properties of the Cdots are summarized in Figure 5. Figure 5A compares the two-photon excitation (TPExc) spectra with the one-photon excitation (OPExc) spectra, and Figure 5B shows the excitation-wavelength dependence of the emission spectra upon one-photon and two-photon excitation at the same total excitation energy (3.4-2.5 eV). There are two remarkable features in the nonlinear spectroscopic data: 1) the TPExc spectrum is blue-shifted from the OPExc spectrum collected at the emission maxima (Fig. 5A), and 2) the Cdots show a nearly excitation-independent emission peaking at $550 \mathrm{~nm}$ upon two-photon excitation in the $700-1000 \mathrm{~nm}$ region (Fig. 5B). In the following discussion, it will be shown that the absorption and emission centered at functionalized $\mathrm{sp}^{2}$ clusters embedded in the basal plane can be responsible for these two features.

For molecular systems, the two-photon excitation response can be rationalized on the basis of the two-photon absorption probability, $\sigma_{2}(\omega)$, given by equation (3): ${ }^{49}$

$$
\sigma_{2}(\omega) \propto\left\langle\left|\boldsymbol{M}_{f g}^{(2)}\right|^{2}\right\rangle \propto\left[\left|\frac{\langle f|\vec{\mu}| k\rangle\langle k|\vec{\mu}| g\rangle}{\omega_{k g}-\omega}\right| \pm\left|\frac{\Delta \vec{\mu}_{f g}\langle f|\vec{\mu}| g\rangle}{\omega}\right|\right]^{2}
$$

where $\boldsymbol{M}_{f g}^{(2)}$ is the two-photon transition matrix element, $\omega$ is the photon energy in a degenerated two-photon absorption process, $g$ is the ground state, $k$ and $f$ are excited states, $\Delta \vec{\mu}$ is the difference between the permanent dipole moment and $\langle i|\vec{\mu}| j\rangle$ is the transition dipole moment between states $i$ and $j$. In dipolar molecules, $\sigma_{2}(\omega)$ is largely determined by the so called dipolar term that contains the product between the difference in the permanent dipole moment $\left(\Delta \vec{\mu}_{f g}\right)$ between the ground and the final 
excited states and the corresponding transition dipole moment weighted by the frequency of the excitation photon $(\omega)$. A similar blueshift between the two-photon and one-photon excitation spectra has been recently reported for a distorted nanographene molecule edge-functionalized with donor and acceptor groups to have a push-pull arrangement favorable to two-photon interactions. ${ }^{50}$ In such system, the lowest energy transitions were shown to be $\pi-\pi^{*}$ transitions localized within the $\mathrm{sp}^{2}$ core with a small variation in the permanent dipole moment and, consequently, a small TPA crosssections. ${ }^{50}$ Higher energy transitions involving charge-transfer between different types of edge functional groups and the $\mathrm{sp}^{2}$ core were more likely to make a significant contribution to the TPA cross-section. This effect resulted in a blueshift between the linear and nonlinear absorption spectra in the push-pull nanographene. ${ }^{50}$ Thus, the observed blueshift in the Cdots supports the existence of $\mathrm{sp}^{2}$ clusters (planar or slightly distorted) bearing a push-pull geometry due to the presence of electron donor amines and electron acceptor carboxylic groups on the edge. 

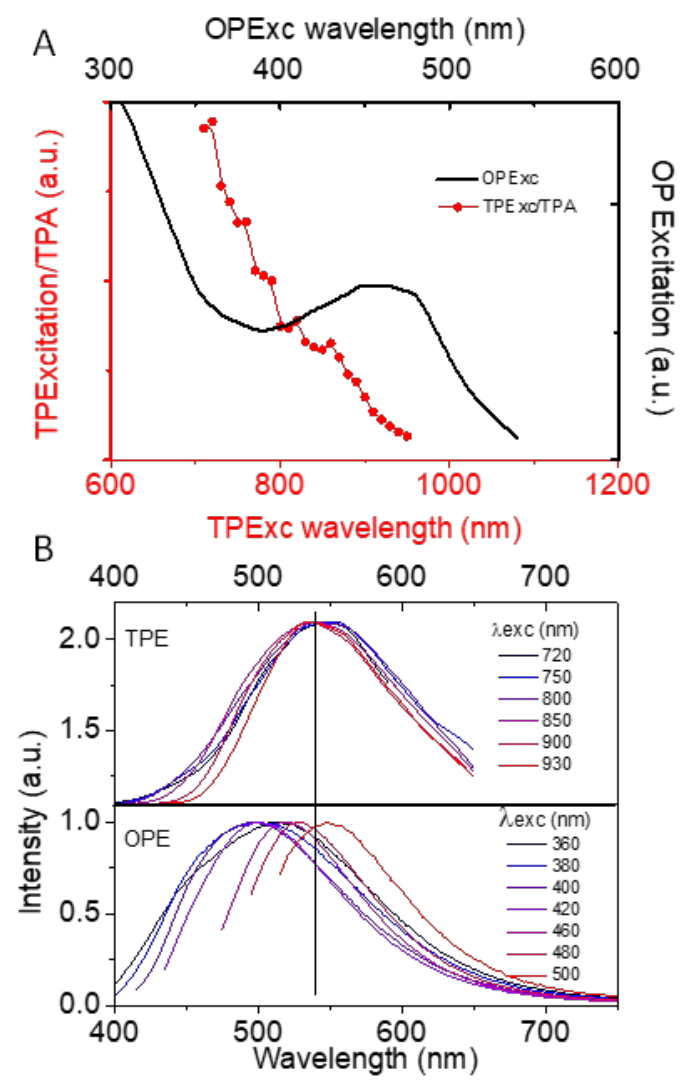

Fig. 5 Two-photon spectroscopic properties of the Cdots. (A) Two-photon excitation spectra (TPEx) compared with the one photon excitation spectra, both collected at 550 nm (OPExc); (B) Excitation dependence of two-photon (TPE) and one-photon (OPE) induced emission at the same total excitation energy.

Conversely to the one-photon excitation, both the emission maximum and the FWHM of the emission band are relatively insensitive to the two-photon excitation energy (Figures 3D and 3E). The TPE maximum changes by $15 \mathrm{~nm}$ while the onephoton emission (OPE) maximum changes by twice as much within the same interval of excitation energies (TPE excited at 720-930 $\mathrm{nm}$ and OPE excited at 360-460 nm). The strongest TPE centered at $550 \mathrm{~nm}$ is clearly red-shifted from the strongest OPE centered at $510 \mathrm{~nm}$. The relatively large and distorted graphene nanoribbon (lateral dimensions of $1 \times 1.9 \mathrm{~nm}$ ) previously mentioned was reported to have an emission band centered at similar wavelengths $(560 \mathrm{~nm}) .{ }^{50}$ The excitation independence of the TPE spectrum can 
be understood on the basis of the selective excitation of push-pull $\mathrm{sp}^{2}$ clusters bearing an edge functionalization that is particularly favorable for nonlinear interaction (e.g with electron donor and electron acceptor groups on opposite ends of the cluster) .

The two-photon absorption cross-section estimated at $720 \mathrm{~nm}$ is $130 \mathrm{GM}$. This is a modest value when compared with the values around $10^{4} \mathrm{GM}$ reported earlier (Table S1). ${ }^{\text {7, 20, 51, } 52}$ However, Cdots with no two-photon emission have also been produced by either top-down or bottom-up methods. ${ }^{16}$ The biggest source of error in the estimation of the TPA cross-section of Cdots using the two-photon stimulated emission method is the concentration of the fluorophore ( $C$ in equation 1$)$. This is a relative method that can only be used to estimate the two-photon absorption cross-section of emissive material. As it can be inferred from the mismatch between the one-photon absorption and onephoton excitation spectra, the heterogeneous mixture of dots contains both emissive and non-emissive materials from which the concentration of emissive material could not be isolated. Nevertheless, the overestimation of the concentration of the emissive material is to some extent compensated by the underestimation of the emission quantum yield ( $\square$ $=$ number of absorbed photons/number of emitted photons) due to the photons absorbed by non-emissive material. Indeed, the value of $130 \mathrm{~nm}$ GM is in good agreement with the TPA cross-section of nanographene molecules produced from controlled step-bystep organic synthesis (also $130 \mathrm{GM}$ ). ${ }^{50}$ Thus, each Cdot does not seem bear more than one of such extended $\mathrm{sp}^{2}$ clusters that are preferentially excited by simultaneous interaction with two NIR photon.

\subsection{Cellular internalization of Cdots}

Since most of the effort in controlling the two-photon induced emission in Cdots relates to their potential applications in biological media, the internalization of Cdots in cells was also investigated. To this end cancer (MCF7 breast cancer cell line) and non-cancer 
cell lines (HEK293T, human embryonic kidney cells) were incubated with Cdots and their distribution pattern was evaluated. The internalization kinetics and distribution obtained was similar in cancer and non-cancer cell lines. To clearly distinguish the Cdots emission from the autofluorescence of living cells, cells were incubated overnight with a solution containing 35 $\square \mathrm{g} / \mathrm{ml}$ of Cdots. In Figure 6 the emission of the Cdots within the cells is compared with that of unlabeled cells (control sample). The fluorescence microscopy images collected using the same acquisition parameters for the Cdot-labeled and unlabeled cells show that Cdots were internalized by the cells and their emission is much stronger than cell autofluorescence. This is true for both linear excitation at $488 \mathrm{~nm}(\mathrm{~A}$ and $\mathrm{C}$ ) and nonlinear excitation at $800 \mathrm{~nm}$ (B and D). With shorter incubation time (15 min, data not shown) no cellular uptake of Cdots was observed. Thus, the specific mechanism of Cdots internalization should be dependent on the activation of endocytic pathways as it required longer incubation times (overnight incubation). Cdots are possibly internalized primarily through caveolae-mediated endocytosis in accordance with observations made in MCF7 cell lines. ${ }^{53}$ In separate experiments, upon incubation of the Cdots overnight, the cells were additionally labeled with plasma membrane (WGA-Alexa 594) and nucleus (Hoescht) specific dyes. The multichannel image in Figure 7 reveal that the Cdots are not adsorbed into cell plasma membrane. The endogenous Cdots fluorescence signal exhibited a vesicular appearance confined to cell cytoplasm further suggesting the potential cellular uptake of Cdots by endocytosis. 


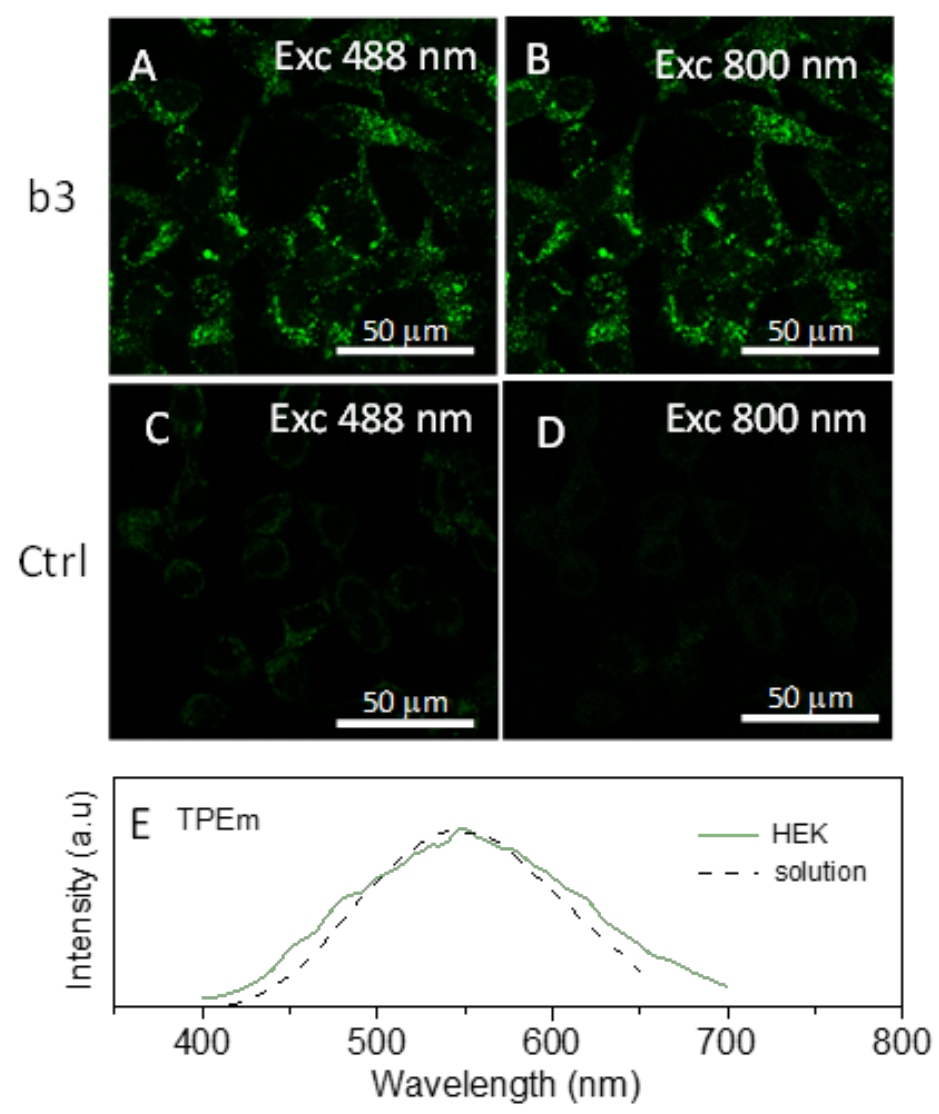

Fig. 6 Fluorescence microscopy images of MCF7 cells incubated overnight with Cdots (A and B) showing that there was internalization of the dots, and control cells (Ctrl) incubated without any fluorescent label (C and D). A and C where collected under linear excitation at $488 \mathrm{~nm}$ while $\mathrm{B}$ and $\mathrm{D}$ were collected in multiphoton mode under $800 \mathrm{~nm}$ excitation. The emission bandwith collected was 470-600 nm for linear excitation and 400-570 nm for multiphoton excitation. Plot E compares the emission spectrum in solution with that recorded for the dots inside the cells. 

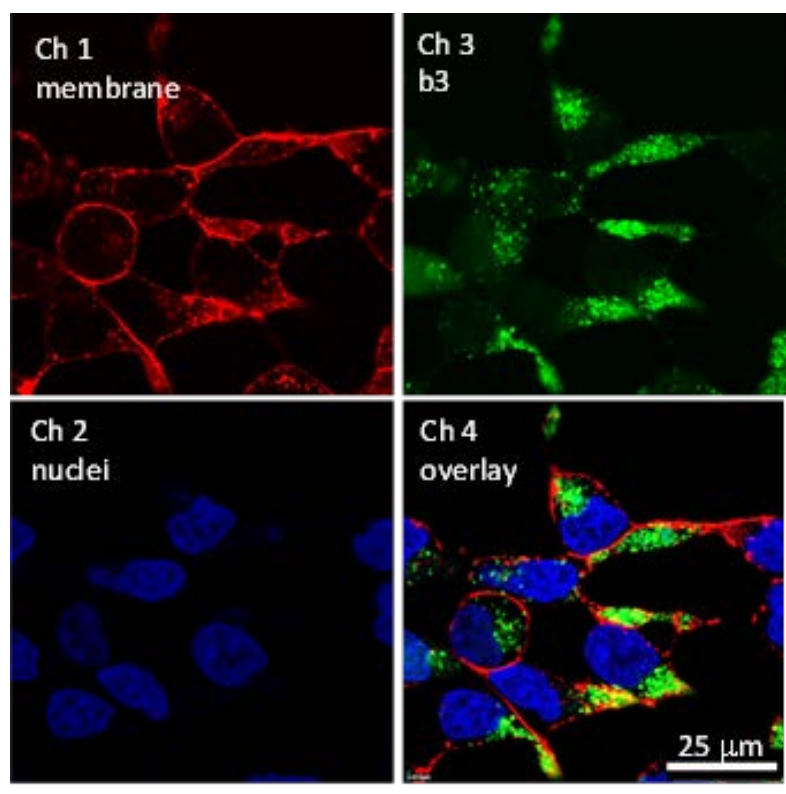

Fig. 7 Multiphoton microscopy images of the distribution of the Cdots inside HEK cells showing that the dots accumulate in the cytoplasm. The membrane marker is shown in red in channel 1, the nuclei marker is shown in blue in channels 2, the dots are shown in green in channel 3 and channel 4 is the overlay of all three channels.

\section{Conclusions}

We presented a comprehensive study of the nonlinear optical properties of nitrogendoped Cdots. The Cdots produced by chemical nanocutting of GO in the presence of hydrogen peroxide and ammonia showed most of the typical chemical, structural and linear spectroscopic features of the carbogenic dots previously reported in the literature. In particular, they were characterized by an excitation dependent emission, with the emission spectrum shifting to longer wavelengths and decreasing in intensity as the excitation energy decreases. Underneath this spectral dispersion, a mirror image relationship between the photoluminescence excitation and the emission spectra was revealed, supporting the localization of the absorption and emission process in isolated emitting sites, and ruling out inter-site charge or energy transfer effects. The emitting sites have been identified as $\mathrm{sp}^{2}$ clusters. The lack of vibronic structure in the absorption 
and emission of such clusters is explained by the $\pi$ - $\pi$ stacking leading to the formation of dimers. The overall spectral dispersion was discussed based on the heterogeneity of the chemical and structural environment (e.g. distortion from planarity induced by defects and the presence of amine groups, cluster size and shape). The nonlinear excitation spectra were discussed in terms of selective two-photon absorption by specific clusters possibly associated with the presence of electron donor and acceptor groups on the edge of the cluster in a push-pull geometry. Finally, we demonstrated that Cdots can be internalized in both healthy and cancer cell lines. The cellular uptake of Cdots appears to be time dependent and related with the activation of endocytic pathways.

\section{Conflicts of interest}

There are no conflicts to declare.

\section{Acknowledgements}

Authors are grateful to Fundação para a Ciência e Tecnologia (FCT, Portugal), European Union, QREN, FEDER and COMPETE for funding the QOPNA, TEMA, and CQFM research unit (project Pest-C/QUI/UI0062/2013, UID/EMS/00481/2013 and

UID/NAN/50024/2013). FCT is acknowledged for the Post-Doctoral grants of C.I.M.S., S.N.P and I.F.A.M., and for supporting the individual work contract of E.M., I. B. and P.A.A.P.M within the Investigador FCT program (SFRH/BPD/105478/2014, SFRH/BPD/92409/2013, SFRH/BPD/ $75782 / 2011$ IF/00759/2013, IF/00582/2015 and IF/00917 /2013). G.G. gratefully acknowledges the funding by European Commission 
under individual fellowship Marie Sklodowska-Curie (NANOTER, Grant Agreement 708351).

\section{References}

1. R. Wang, K. Q. Lu, Z. R. Tang and Y. J. Xu, J. Mater. Chem. A, 2017, 5, 37173734.

2. R. B. Xie, Z. F. Wang, W. Zhou, Y. T. Liu, L. Z. Fan, Y. C. Li and X. H. Li, Anal Methods-Uk, 2016, 8, 4001-4016.

3. Z. L. Peng, X. Han, S. H. Li, A. O. Al-Youbi, A. S. Bashammakh, M. S. ElShahawi and R. M. Leblanc, Coord. Chem. Rev., 2017, 343, 256-277.

4. S. Y. Lu, L. Z. Sui, J. J. Liu, S. J. Zhu, A. M. Chen, M. X. Jin and B. Yang, Advanced Materials, 2017, 29.

5. H. J. Sun, L. Wu, W. L. Wei and X. G. Qu, Mater Today, 2013, 16, 433-442.

6. J. L. Li, B. Tang, B. Yuan, L. Sun and X. G. Wang, Biomaterials, 2013, 34, 9519-9534.

7. Q. Liu, B. D. Guo, Z. Y. Rao, B. H. Zhang and J. R. Gong, Nano Lett., 2013, 13, 2436-2441.

8. W. R. Zipfel, R. M. Williams and W. W. Webb, Nat Biotechnol, 2003, 21, 13681376.

9. L. Guo and M. S. Wong, Adv. Mat., 2014, 26, 5400-5428.

10. G. S. He, L. S. Tan, Q. Zheng and P. N. Prasad, Chem Rev, 2008, 108, 12451330.

11. I. F. A. Mariz, E. M. S. Macoas, J. M. G. Martinho, L. Zou, P. C. Zhou, X. G. Chen and J. G. Qin, J Mater Chem B, 2013, 1, 2169-2177.

12. E. Maçoas, G. Marcelo, S. Pinto, T. Caneque, A. M. Cuadro, J. J. Vaquero and J. M. G. Martinho, Chem. Commun., 2011, 47, 7374-7376. 
13. R. J. Gui, J. Hui, Z. H. Wang and L. J. Tan, Coord. Chem. Rev., 2017, 338, 141-185.

14. C. F. Wang, X. Wu, X. P. Li, W. T. Wang, L. Z. Wang, M. Gu and Q. Li, Journal of Materials Chemistry, 2012, 22, 15522-15525.

15. J. Qian, D. Wang, F. H. Cai, W. Xi, L. Peng, Z. F. Zhu, H. He, M. L. Hu and S. L. He, Angewandte Chemie-International Edition, 2012, 51, 10570-10575.

16. X. M. Wen, P. Yu, Y. R. Toh, X. Q. Ma and J. Tang, Chemical Communications, 2014, 50, 4703-4706.

17. J. Lin, X. Y. Chen and P. Huang, Advanced Drug Delivery Reviews, 2016, 105, 242-254.

18. K. L. Schroeder, R. V. Goreham and T. Nann, Pharm Res-Dordr, 2016, 33, 2337-2357.

19. H. Jin, R. J. Gui, Y. F. Wang and J. Sun, Talanta, 2017, 169, 141-148.

20. H. D. Ha, M. H. Jang, F. Liu, Y. H. Cho and T. S. Seo, Carbon, 2015, 81, 367-375.

21. R. J. Gui, H. Jin, Y. F. Wang and J. Sun, Sensors and Actuators B-Chemical, 2017, 245, 386-394.

22. H. Wang, J. Di, Y. B. Sun, J. P. Fu, Z. Y. Wei, H. Matsui, A. D. Alonso and S. Q. Zhou, Advanced Functional Materials, 2015, 25, 5537-5547.

23. S. J. Zhu, Y. B. Song, X. H. Zhao, J. R. Shao, J. H. Zhang and B. Yang, Nano Res., 2015, 8, 355-381.

24. Z. X. Gan, H. Xu and Y. L. Hao, Nanoscale, 2016, 8, 7794-7807.

25. M. A. Sk, A. Ananthanarayanan, L. Huang, K. H. Lim and P. Chen, J Mater Chem C, 2014, 2, 6954-6960. 
26. S. Ghosh, A. M. Chizhik, N. Karedla, M. O. Dekaliuk, I. Gregor, H. Schuhmann, M. Seibt, K. Bodensiek, I. A. T. Schaap, O. Schulz, A. P. Demchenko, J. Enderlein and A. I. Chizhik, Nano Letters, 2014, 14, 5656-5661.

27. D. Kozawa, Y. Miyauchi, S. Mouri and K. Matsuda, J Phys Chem Lett, 2013, 4, 2035-2040.

28. H. Park, S. H. Noh, J. H. Lee, W. J. Lee, J. Y. Jaung, S. G. Lee and T. H. Han, Scientific Reports, 2015, 5.

29. J. R. Lakowicz, Principles of Fluorescence Spectroscopy, Springer, Baltimore, USA, Third edn., 2006.

30. S. de Reguardati, J. Pahapill, A. Mikhailov, Y. Stepanenko and A. Rebane, Optics Express, 2016, 24, 9053-9066.

31. C. Xu and W. W. Webb, J. Opt. Soc. Am. B, 1996, 13, 481-491.

32. H. Tetsuka, R. Asahi, A. Nagoya, K. Okamoto, I. Tajima, R. Ohta and A. Okamoto, Advanced Materials, 2012, 24, 5333-5338.

33. V. Tucureanu, A. Matei and A. M. Avram, Critical Reviews in Analytical Chemistry, 2016, 46, 502-520.

34. S. J. Zhu, J. H. Zhang, S. J. Tang, C. Y. Qiao, L. Wang, H. Y. Wang, X. Liu, B. Li, Y. F. Li, W. L. Yu, X. F. Wang, H. C. Sun and B. Yang, Advanced Functional Materials, 2012, 22, 4732-4740.

35. F. Tuinstra and J. L. Koenig, Journal of Chemical Physics, 1970, 53, 1126$\&$.

36. A. C. Ferrari and J. Robertson, Phys Rev B, 2000, 61, 14095-14107.

37. S. J. Zhu, J. H. Zhang, C. Y. Qiao, S. J. Tang, Y. F. Li, W. J. Yuan, B. Li, L. Tian, F. Liu, R. Hu, H. N. Gao, H. T. Wei, H. Zhang, H. C. Sun and B. Yang, Chemical Communications, 2011, 47, 6858-6860. 
38. L. L. Li, J. Ji, R. Fei, C. Z. Wang, Q. Lu, J. R. Zhang, L. P. Jiang and J. J. Zhu, Advanced Functional Materials, 2012, 22, 2971-2979.

39. D. Y. Pan, L. Guo, J. C. Zhang, C. Xi, Q. Xue, H. Huang, J. H. Li, Z. W. Zhang, W. J. Yu, Z. W. Chen, Z. Li and M. H. Wu, Journal of Materials Chemistry, 2012, 22, 3314-3318.

40. J. Peng, W. Gao, B. K. Gupta, Z. Liu, R. Romero-Aburto, L. H. Ge, L. Song, L. B. Alemany, X. B. Zhan, G. H. Gao, S. A. Vithayathil, B. A. Kaipparettu, A. A. Marti, T. Hayashi, J. J. Zhu and P. M. Ajayan, Nano Letters, 2012, 12, 844849.

41. F. Jiang, D. Q. Chen, R. M. Li, Y. C. Wang, G. Q. Zhang, S. M. Li, J. P. Zheng, N. Y. Huang, Y. Gu, C. R. Wang and C. Y. Shu, Nanoscale, 2013, 5, 1137-1142.

42. B. Stevens, Spectrochim Acta, 1962, 18, 439-448.

43. X. X. Deng, J. Sun, S. W. Yang, H. Shen, W. Zhou, J. Lu, G. Q. Ding and Z. Y. Wang, Appl Phys Lett, 2015, 107.

44. M. Fu, F. Ehrat, Y. Wang, K. Z. Milowska, C. Reckmeier, A. L. Rogach, J. K. Stolarczyk, A. S. Urban and J. Feldmann, Nano Letters, 2015, 15, 6030-6035.

45. S. J. Zhu, X. H. Zhao, Y. B. Song, S. Y. Lu and B. Yang, Nano Today, 2016, 11, 128-132.

46. J. Schneider, C. J. Reckmeier, Y. Xiong, M. von Seckendorff, A. S. Susha, P. Kasak and A. L. Rogach, Journal of Physical Chemistry C, 2017, 121, 20142022.

47. Y. Xiong, J. Schneider, C. J. Reckmeier, H. Huang, P. Kasak and A. L. Rogach, Nanoscale, 2017, 9, 11730-11738.

48. F. P. Schwarz and S. P. Wasik, Anal Chem, 1976, 48, 524-528. 
49. K. Kamada, Y. Iwase, K. Sakai, K. Kondo and K. Ohta, J. Phys. Chem. C, 2009, 113, 11469-11474.

50. C. M. Cruz, I. R. Marquez, I. F. A. Mariz, V. Blanco, C. Sanchez-Sanchez, J. M. Sobrado, J. A. Martin-Gago, J. M. Cuerva, E. Macoas and A. G. Campana, Chemical Science, 2018.

51. L. Cao, X. Wang, M. J. Meziani, F. S. Lu, H. F. Wang, P. J. G. Luo, Y. Lin, B. A. Harruff, L. M. Veca, D. Murray, S. Y. Xie and Y. P. Sun, Journal of the American Chemical Society, 2007, 129, 11318-+.

52. Y. L. Shi, A. Pramanik, C. Tchounwou, F. Pedraza, R. A. Crouch, S. R. Chavva, A. Vangara, S. S. Sinha, S. Jones, D. Sardar, C. Hawker and P. C. Ray, ACS Appl. Mater. Interfaces, 2015, 7, 10935-10943.

53. C. Y. Wu, C. Wang, T. Han, X. J. Zhou, S. W. Guo and J. Y. Zhang, Advanced Healthcare Materials, 2013, 2, 1613-1619. 